\title{
Isoapariencia y el método del queso suizo, un factor de riesgo durante la práctica profesional en enfermería
}

\author{
Gabriela Viviana Rodríguez Zamora \\ Gabivivi121999@gmail.com
}

Lic. Eulalia Analuisa

ei.analuisa@uta.edu.ec

Ambato - Ecuador

\section{RESUMEN}

Objetivo: Analizar la isoapariencia y el método del queso suizo, durante la práctica profesional en enfermería Método: Se realizó mediante un estudio cuantitativo de tipo transversal y analítico las personas encuestadas fueron profesionales de Enfermería del Hospital General Docente Ambato. Para la recolección de los datos se utilizó Google Docs que es un procesador de texto en línea y el software SPSS para el análisis de los datos recopilados. El cuestionario diseñado ad hoc incluía 27 ítems con cinco posibles respuestas según escala Likert ( $1=$ muy en desacuerdo a 5= muy de acuerdo). Resultados: Se realizó un estudio donde los profesionales consideraron que la isoapariencia es un factor de riesgo durante la práctica profesional en donde al menos un $15.40 \%$ de los sujetos han cometido algún error de medicación. Conclusión: La isoapariencia es apreciada como el principal factor de riesgo durante la práctica profesional esto se puedo evidenciar al emplear el modelo del queso suizo el mismo que ayudo a identificar posibles fallas en el sistema de salud.

Palabras clave: enfermería; farmacovigilancia; errores de medicación; isoapariencia farmacéutica. 


\title{
Isoappearance and the swiss cheese method, a risk factor during nursing practice
}

\begin{abstract}
Objective: To analyze the isoapparency and the Swiss cheese method during professional nursing practice. Method: A quantitative, cross-sectional and analytical study was carried out. The persons surveyed were nursing professionals from the Hospital General Docente Ambato. Google Docs, which is an online word processor, was used for data collection and SPSS software was used to analyze the data collected. The ad hoc designed questionnaire included 27 items with five possible answers according to Likert scale (1= strongly disagree to $5=$ strongly agree). Results: A study was conducted where professionals considered isoapparency as a risk factor during professional practice where at least $15.40 \%$ of the subjects have committed some medication error. Conclusion: Isoapparency is appreciated as the main risk factor during professional practice. This was evidenced by the use of the Swiss cheese model, which helped to identify possible failures in the health system.
\end{abstract}

Key words: nursing; pharmacovigilance; medication errors; pharmaceutical isoapparency.

Artículo recibido: 30 noviembre. 2021 Aceptado para publicación: 29 diciembre 2021 Correspondencia: Gabivivi121999@gmail.com

Conflictos de Interés: Ninguna que declarar 


\section{OBJETIVOS}

\subsection{Objetivo principal}

- Determinar la isoapariencia y el método del queso suizo, durante la práctica profesional en enfermería

\section{OBJETIVOS SECUENDARIOS}

\subsection{Objetivo secundario}

- Determinar las áreas de mayor relevancia en relación a la isoapariencia durante la práctica del profesional de enfermería.

- Establecer complicaciones relacionadas con la isoapariencia en la práctica diaria en el personal de enfermería.

- Describir los factores relacionados a la isoapariencia y fallo del sistema en base del modelo queso suizo

\section{INTRODUCCIÓN}

La finalidad de la enfermería es ofrecer cuidados de calidad y calidez, respetando los derechos, valores, creencias y costumbres de todas las personas, fomentando la seguridad del paciente. Una de las funciones de nuestra profesión es la administración de medicamentos.(Palacio Lapuente, 2017) Por lo cual a lo largo de nuestra carrera profesional es fundamental adquirir habilidades y conocimiento sobre los fármacos.(Pérez, 2019) Sin embargo, existen situaciones en donde la calidad de dicha función se ve afectada de manera negativa, a causa de errores, incidentes y confusiones una de ellas es la similitud existente entre los medicamentos (isoapariencia) lo cual ponen en riesgo la seguridad y bienestar de los pacientes. (Ingidua et al., 2020). Por lo cual se realiza un estudio cuantitativo de tipo comparativo con la finalidad de concentrar en un solo documento toda la información que existe sobre los errores durante la administración de medicamentos dado que tanto la imagen de los medicamentos, carga laboral y utilización de las practicas seguras son factores que no pueden ser controlados por el profesional de Enfermería, esto puede exponer la seguridad del usuario y del profesional desde el punto de vista legal.(Arguin, s. f.) Sin embargo, los sistemas para la prevención de errores de medicación, basados en el modelo del queso suizo de James Reason postulan que estos errores deben ser analizados como fallos del sistema y no como errores individuales. Además, son inherentes a la naturaleza humana, independientemente de la capacitación del sujeto para desempeñar la tarea, asimismo la sobrecarga laboral 
conduciría a fracasos laborales y personales, poniendo en juego el bienestar del paciente lo cual conlleva a una gran confusión y posibles consecuencias. Estos errores pueden provocar desde alteraciones leves a resultados fatales, según el fármaco que actúe. (Feliu, 2018).Existen evidencias científicas sobre la isoapariencia en la administración de medicamentos, la Organización Mundial de la Salud emitió un comunicado en donde dan a conocer que varios niños menores de 2 años habían muerto en una campaña de vacunación de sarampión y rubeola (2016) que se estaba llevando a cabo en el norte de Siria, como consecuencia de un error de medicación.(Monise, 2019) Las vacunas se reconstituyeron equivocadamente con ampollas que contenían el relajante neuromuscular atracurio, en lugar de usar las ampollas de diluyente de las vacunas, se confundieron con las del disolvente por tener un aspecto similar. De 75 niños que recibieron la vacuna, al menos 15 fallecieron.(Mr et al., 2016).A lo largo de la historia los errores durante la administración de medicación ha sido un tema de estudio, esto puede ser a causa de la gran variedad de medicamentos existes en el ámbito hospitalario al tratar de analizar la causa que han desencadenado dichos errores, se pueden encontrar el incumplimiento de los "cinco correctos", las elevadas cargas de trabajo, el almacenamiento incorrecto de los fármacos y la similitud en los envases de medicamentos con principios activos diferentes a este último se ha denominado como "isoapariencia farmacéutica" o "Look Alike Drugs"(Cassius et al., 2019)( sustancias similares, (parecidas) a las drogas) en las bibliografías internacional (Ingidua et al., 2020). Su comercialización y producción de las distintas casas farmacéuticas que presentan una apariencia parecida de nombre similar con diferentes principios activos ocasionan errores en la práctica profesional, estos también pueden incluir fallos en la prescripción, etiquetado, preparación, vía de administración y dosis.(Arguin, s. f.).El diseño del medicamento como la caja, la forma, el tamaño o color son una guía para los profesionales que administran los medicamentos, por esta razón se originan confusiones en casos de situaciones urgentes, medicamentos tales como la adrenalina atropina, naloxona, dobutamina isoproterenol o haloperidol qué son ampollas del mismo color y tamaños muy similares que se ubican en lugares de gestión de emergencia como ambulancias quirófanos e incluso UCI pueden afectar a la seguridad del paciente.(Palacio Lapuente, 2017).Existen instituciones y movimientos que tratan de promover la seguridad del paciente entre ellos encontramos al instituto para el uso seguro de los medicamentos (ISMP) (ISMP España, s. f.)quien propone medidas para 
evitar la confusión de medicamentos en donde podemos encontrar información tales como recomendaciones de prácticas seguras en la conciliación de la medicación al alta hospitalaria en pacientes crónicos, prevención de errores causados por confusión en los nombres de los medicamentos, prevención de errores causados por el etiquetado y envasado de los medicamentos.(Isoapariencia para minimizar los errores en la dispensación de medicamentos - iSanidad, s. f.).En ocasiones los errores no suelen aprovecharse como una oportunidad para analizar los fallos y es aquí donde encontramos el MODELO DEL QUESO SUIZO propuesto por James Reaso expli por qué ocurren fallas, accidentes y desastres identificando el error en todo el sistema, una de la ideas de Reason fue que los seres humanos son propensos a errores operativos, los cuales requieren sistemas que estén diseñados adecuadamente para mitigar estos riesgos.(12) Según el modelo casi todos los eventos adversos ocurren debido a la combinación de dos factores: fallas activas y latentes que dan origen al error.(Monise, 2019)

No obstante, estas condiciones se pueden identificar y remediar antes de que ocurra un evento adverso, mediante simulaciones, análisis de escenarios, conduciendo una gestión proactiva del riesgo.(Feliu, 2018)

Por ello, es importante poseer un conocimiento amplio acerca de dichos errores, así como las estrategias de prevención y actuación ante los mismos, sin olvidar la importancia de notificarlos para realizar un análisis exhaustivo de las causas y los factores que los han desencadenado; por ello la importancia de este trabajo de investigación radica en ayudar a retomar y reforzar las actividades de los profesionales de enfermería para evitar dichos errores. (Sánchez Ballesteros \& Montero, 2016)

\section{ESTRATEGIAS METODOLÓGICAS O MATERIALES Y MÉTODOS}

La investigación se realizará mediante un estudio cuantitativo de tipo transversal y analítico en donde se realizará una búsqueda de documentos científicos teniendo en cuenta su nivel de evidencia científica y grado de recomendación. (A systematic literature review on strategies to avoid look-alike errors of labels $\mid$ SpringerLink, s. f.).La población fue de 305 profesionales de enfermería del Hospital General Docente Ambato equivalente al $100 \%$ de la población. Se distribuyeron un total de 305 cuestionarios de forma online en la plataforma de google docs (formulario).

El instrumento utilizado para recoger las opiniones de los profesionales de Enfermería fue diseñado ad hoc. Las preguntas del cuestionario se diseñaron con la intención de 
conocer si es frecuente que los profesionales de enfermería trabajen con este tipo de envases, en qué circunstancias consideraban más peligrosa la similitud en el envasado, este problema dio lugar a un error de administración.

El cuestionario diseñado conteniente 27 preguntas, con cinco opciones de respuesta: muy en desacuerdo, poco de acuerdo, indiferente, bastante de acuerdo, muy de acuerdo. Además, se utilizó el software SPSS para realizar el análisis estadístico y recolección de datos para crear tablas y gráficas.

Para la realización del estudio se solicitó la autorización del Hospital General Docente Ambato, siendo favorable para dicho propósito. El estudio fue aprobado por la Comisión de Investigación de la Universidad Técnica De Ambato, siendo voluntaria la participación del estudio.

\section{Contenido}

El modelo del queso suizo es utilizado para explicar porque se generan los accidentes, desastres y fallas en los sistemas a partir de los errores humanos creando un análisis para prevenir los riesgos. La estructura propuesta por James Reason explica que cualquier elemento de una organización puede considerarse un segmento de queso, a pesar de que estos elementos no sean perfectos ya que contienen defectos y debilidades, estos componentes se representa como agujeros es decir si estos errores se alinean permiten crear una trayectoria de oportunidad para que se genere un accidente(Pinheiro et al., 2017).

Según el modelo de Reason, cuantas más capas de intervenciones efectivas se implementen, es menos probable que exista un fallo, algunas ideas que condujeron a la construcción de esta teoría integrada llamada queso suizo nos menciona que los accidentes son causados por la casualidad o afinidad de múltiples factores que en ocasiones están alineados pero inactivos, esperando un desencadenante en cualquier momento, además estos factores pueden variar desde actos individuales hasta errores organizativos.(SciELO - Brasil - Risk factors for medication errors in the electronic and manual prescription Risk factors for medication errors in the electronic and manual prescription, s. f.) .Según el modelo, casi todos los eventos adversos ocurren debido a la combinación de dos factores: fallas activas como no usar el equipo de seguridad, no seguir el procedimiento estándar o cualquier otra actividad que eventualmente se requiera y latentes que comprende una falla incrustada en el proceso, procedimiento y máquinas. 
Estas son fallas que esperan ser accionadas por una falla activa .(«Modelo de queso suizo para el análisis de riesgos y fallas», 2019).Los errores de la medicación se definen como cualquier error que se genere en la utilización de medicamentos. El National Coordinating Council for Medication Error Reporting and Prevention (NCCMERP) define los errores de la medicación como "cualquier incidente prevenible que pueda causar algún daño al paciente o provocar un uso inapropiado de los medicamentos, cuando éstos están bajo el control de los profesionales sanitarios.(Tseng et al., 2018) Estos incidentes pueden estar relacionados tanto como la práctica profesional, con los procedimientos o con los sistemas, incluyendo fallos en la prescripción, comunicación, etiquetado, envasado, denominación, preparación, dispensación, distribución, administración y utilización”.(Villegas et al., 2018) .Si bien es cierto es posible encontrar algunos fármacos isoaparentes (fármacos con apariencia similar) entre sí, este problema es más frecuente dentro del grupo de los medicamentos genéricos debido a que las empresas farmacéuticas ofrecen el mismo principio activo con diferentes aspectos, entre ellas encontramos características como el color, la tipografía, el envase, la forma y el tamaño que componen la imagen del producto.(Suntasig Guaña, 2015) Dado que la apariencia de los medicamentos son factores no controlables por el profesional de Enfermería, pero una administración incorrecta del producto afecta a la seguridad del paciente desde el punto de vista legal. No obstante, los sistemas para la prevención de errores de medicación basados en el modelo del queso suizo y el informe "To Err is Human", postulan que estos errores deben ser analizados como fallos del sistema y no como errores individuales, además son innatos a la naturaleza humana, independientemente de la capacitación del sujeto para desempeñar la tarea.(Palacio et al., 2017).Después de 15 años de la publicación de”To Err is Human" sitúa los acontecimientos adversos como séptima causa de muerte en los Estados Unidos después de una enfermedad cardíaca o cáncer. "To Err is Human" insistía en la necesidad de un cambio en el sistema porque se observaba que era habitual que los profesionales sanitarios consideraran los errores como un signo de incompetencia o imprudencia de la persona, en lugar de aprender para mejorar la seguridad y prevenir nuevos riesgos, el instinto era de ocultarlos por miedo a represalias legales o incluso la pérdida de la propia profesión.(Díez et al., 2017).En septiembre de 2013, un nuevo estudio publicado en el Journal of Patient Safety concluyó que los errores médicos que provocan la muerte de pacientes en hospitales son mucho más numerosos de 
lo que se pensaba anteriormente y por esta causa se podrían haber llegado a producir hasta 440.000 al año.(Montero Delgado et al., 2020)

\section{RESULTADOS Y DISCUSIÓN}

A continuación, se muestran los resultados obtenidos mediante la toma de datos realizados a través de encuestas online basado en el tema "ISOAPARIENCIA Y EL MÉTODO DEL QUESO SUIZO, UN FACTOR DE RIESGO DURANTE LA PRÁCTICA PROFESIONAL EN ENFERMERÍA”, pertenecientes a las diferentes áreas que ofrece el Hospital General Docente Ambato. Donde se evaluaron distintos parámetros tomando en cuenta las preguntas más importantes en relación con el tema mencionado, tomando en cuenta las edades y los servicios en donde se desempeñan dichos profesionales.

El 46.56\% de los profesionales que laboran en el Hospital General Docente Ambato comprenden edades entre 20-30 años entre hombre y mujeres.

Figura 1 Porcentaje de edades

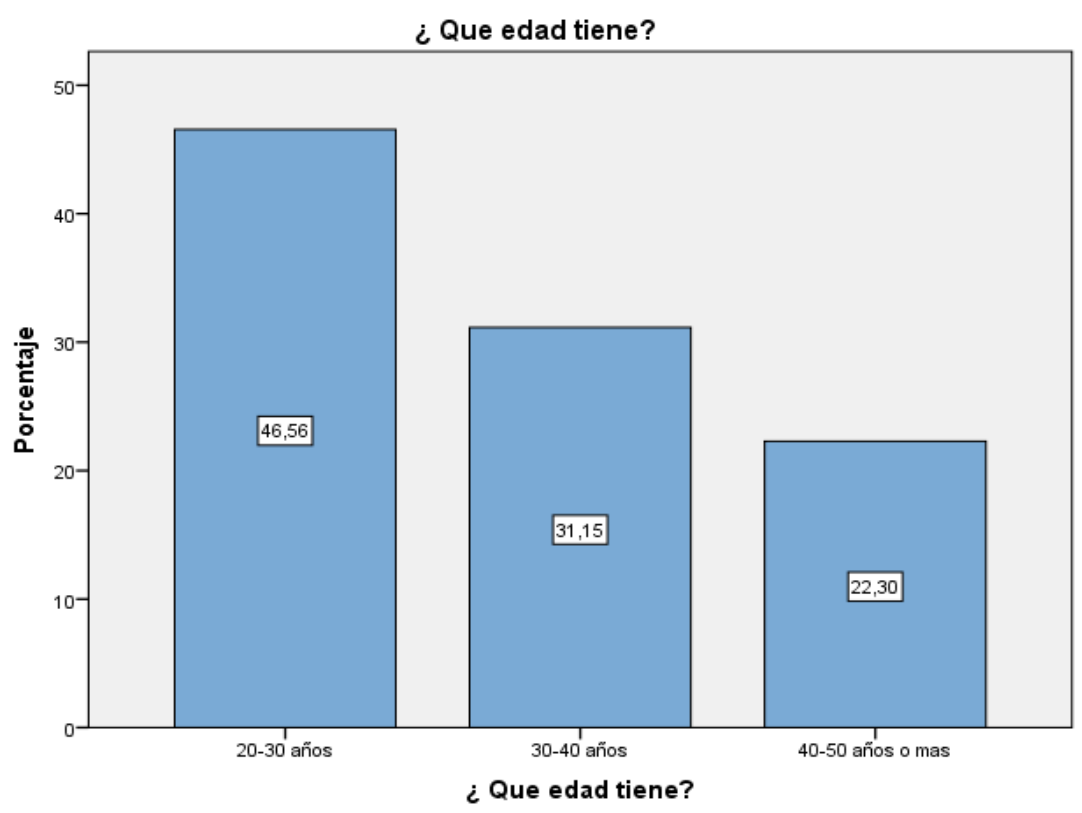

El $14.43 \%$ de la población pertenece al servicio de emergencia siendo el más afectado al presentar un mayor riesgo durante la administración de medicamentos esto se puede visualizar en la figura 2. 
Figura 2 Áreas

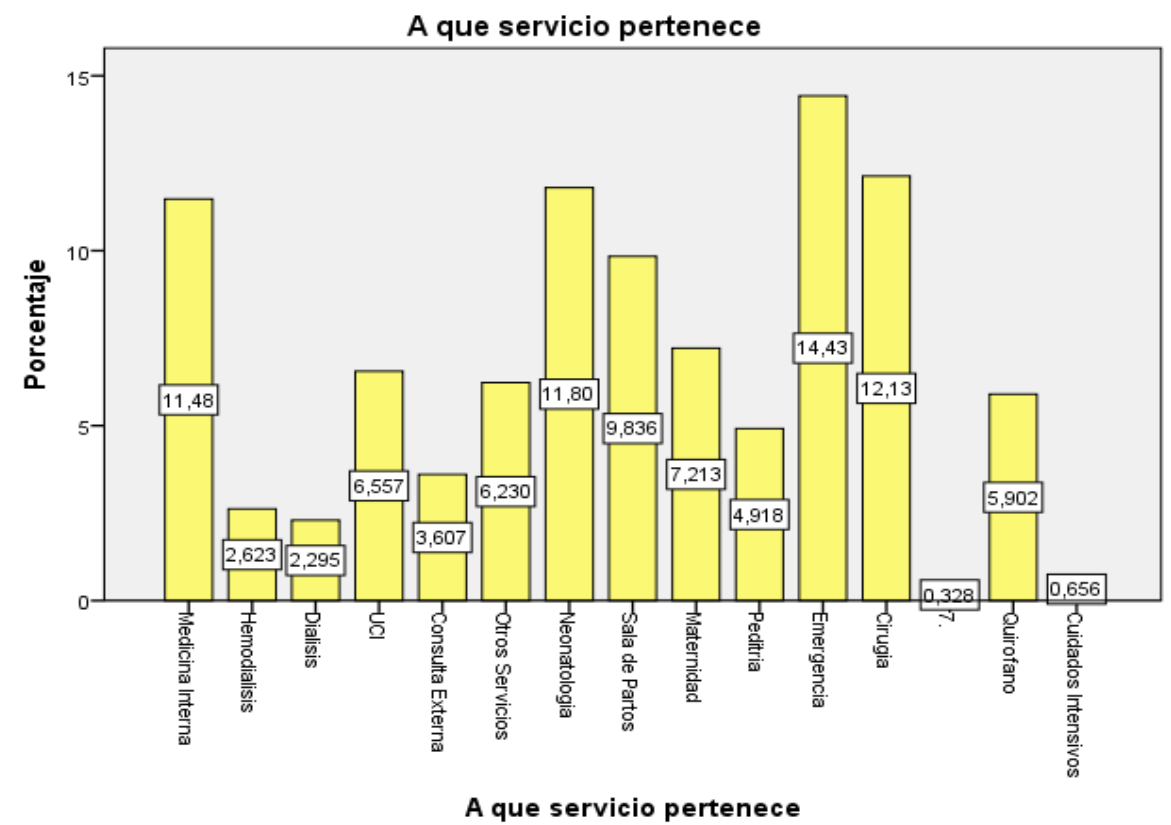

Durante la práctica profesional se pueden encontrar distintos medicamentos con envases muy similares entre sí, que afecta al paciente poniendo en riesgo su salud además de dificultar al experto durante la administración de medicación. Esto se puede observar en la figura 3 en donde el $31.11 \%$ y el $26.56 \%$ de la población afirma que existen medicamentos genéricos con envases muy similares y tan solo el $10.16 \%$ sostiene una opinión diferente. Al existir medicamentos con distinto principios activos y envases, se podría cometer errores así lo afirman los profesionales con un $36,72 \%$ perteneciente al bastante de acuerdo y 37,38\% muy de acuerdo. Medicamentos tales como adrenalina, aminofilina, diazepan (valium), dobutamina (dobutrex), dopamina, fenintoína , lidocaína, que se usa dentro de una emergencia suelen ser confundidas al momento de la atención médica con un $40 \%$ en bastante de acuerdo y con un 33,44\% muy de acuerdo además el $36,07 \%$ de la población testifica que la ausencia de medicamentos isoaparentes facilitaría la práctica profesional e incluso en ocasiones un paciente ha referido no tomar la medicación porque ha cambiado la presentación habitual que consumen normalmente el $29.18 \%$ y $19.34 \%$ pertenece al bastante de acuerdo y muy de acuerdo respectivamente(Errores de medicación en pacientes hospitalizados: una revisión sistemática, s. f.).Según la encuesta se preguntó si en alguna ocasión se ha confundido de medicamento por el parecido con otro, se encontró que el $38.03 \%$ perteneciente al poco de acuerdo y 24,59\% muy desacuerdo, si en alguna ocasión es administrado un fármaco 
erróneo porque su envase era muy parecido al del fármaco prescrito con un $44.59 \%$ pertenece al muy desacuerdo, la mayoría de las persona encuestadas afirman cumplir con la regla de los 5 correctos 2 veces como se recomienda con un $57.05 \%$ muy de acuerdo. En alguna ocasión, un compañero/a me ha confesado haber administrado un fármaco erróneo por su parecido con el fármaco distinto el $20.66 \%$ muy desacuerdo y $27,54 \%$ poco de acuerdo y tan solo el 16,46\% sostiene una opinión diferente. El personal de enfermería conoce y notifica los errores de administración cuando se ha percatado de ellos con 37,05\% bastante de acuerdo y el 28,20\% muy de acuerdo(Lacasa, 2019) (tabla 3).

Tabla 3: preguntas

\begin{tabular}{|l|l|l|l|l|l|}
\hline \multicolumn{1}{|c|}{ Pregunta } & $\begin{array}{c}\text { Muy } \\
\text { desacuerdo }\end{array}$ & $\begin{array}{c}\text { Poco de } \\
\text { acuerdo }\end{array}$ & Indiferente & $\begin{array}{c}\text { Bastante } \\
\text { de } \\
\text { acuerdo }\end{array}$ & $\begin{array}{c}\text { Muy de } \\
\text { acuerdo }\end{array}$ \\
\hline $\begin{array}{l}\text { Es frecuente encontrar en mi } \\
\text { control de enfermería fármacos } \\
\text { genéricos distintos con envases } \\
\text { similares }\end{array}$ & $10,2 \%$ & $22,60 \%$ & $7,50 \%$ & $33,10 \%$ & $26,60 \%$ \\
\hline $\begin{array}{l}\text { Considero peligroso el parecido } \\
\text { entre fármacos genéricos con } \\
\text { distintos principio activo, tanto } \\
\text { para los pacientes como para los } \\
\text { profesionales }\end{array}$ & $10,2 \%$ & $9,50 \%$ & $6,20 \%$ & $36,70 \%$ & $37,40 \%$ \\
\hline $\begin{array}{l}\text { Considero muy peligrosa la } \\
\text { existencia dérmacos } \\
\text { isoaparentes ante la atención de } \\
\text { una emergencia }\end{array}$ & $9,8 \%$ & $11,80 \%$ & $4,90 \%$ & $40 \%$ & $33,40 \%$ \\
\hline $\begin{array}{l}\text { En alguna ocasión he confundido } \\
\text { un fármaco por su parecido con } \\
\text { otro/s }\end{array}$ & $24,6 \%$ & $38 \%$ & $11,80 \%$ & $14,10 \%$ & $11,50 \%$ \\
\hline $\begin{array}{l}\text { En alguna ocasión es } \\
\text { administrado un fármaco erróneo } \\
\text { porque su envase era muy } \\
\text { parecido al del fármaco prescrito }\end{array}$ & $44,6 \%$ & $23,90 \%$ & $11,50 \%$ & $10,50 \%$ & $9,50 \%$ \\
\hline $\begin{array}{l}\text { Cumplo siempre estrictamente la } \\
\text { regla de los 5 correctos } \mathbf{2} \text { veces } \\
\text { cómo se recomienda) }\end{array}$ & $6,2 \%$ & $7,50 \%$ & $3,60 \%$ & $25,60 \%$ & $57 \%$ \\
\hline $\begin{array}{l}\text { Creo que la ausencia de } \\
\text { isoapariencia en fármacos } \\
\text { diferentes facilitaría mi práctica } \\
\text { profesional ques deberían estandarizar }\end{array}$ & $9,8 \%$ & $15,70 \%$ & $9,80 \%$ & $36,10 \%$ & $28,50 \%$ \\
\hline $\begin{array}{l}\text { Creo que } \\
\text { las presentaciones de un mismo } \\
\text { fármaco cuando pertenece a } \\
\text { laboratorios distintos }\end{array}$ & $7,9 \%$ & $8,90 \%$ & $9,50 \%$ & $39,70 \%$ & $34,10 \%$ \\
\hline & & & & & \\
\hline
\end{tabular}




\begin{tabular}{|l|l|l|l|l|l|}
\hline $\begin{array}{l}\text { En alguna ocasión un paciente me } \\
\text { ha referido no tomar la } \\
\text { medicación porque ha cambiado } \\
\text { la presentación habitual } \\
\text { medicamento que consumen } \\
\text { normalmente }\end{array}$ & $14,4 \%$ & $23,60 \%$ & $13,40 \%$ & $29,20 \%$ & $19,30 \%$ \\
\hline $\begin{array}{l}\text { Considero que cambiarán algún } \\
\text { aspecto del de envases diferentes, } \\
\text { como colores o tipografía, podría } \\
\text { ayudarme a diferenciar mejor los } \\
\text { fármacos }\end{array}$ & $6,2 \%$ & $16,10 \%$ & $9,20 \%$ & $38,40 \%$ & $30,20 \%$ \\
\hline $\begin{array}{l}\text { En alguna ocasión, un } \\
\text { compañero/a me ha confesado } \\
\text { haber administrado un fármaco } \\
\text { errores por su parecido con el } \\
\text { fármaco distinto }\end{array}$ & $20,7 \%$ & $27,50 \%$ & $20,30 \%$ & $19 \%$ & $12,50 \%$ \\
\hline $\begin{array}{l}\text { Siempre he notificado los errores } \\
\text { de administración cuando se me } \\
\text { he percatado de ellos }\end{array}$ & $8,5 \%$ & $14,10 \%$ & $12,10 \%$ & $37 \%$ & $28,20 \%$ \\
\hline $\begin{array}{l}\text { En el almacén de medicación en } \\
\text { mi servicio los fármacos se } \\
\text { ordenan por } \\
\text { farmacológica }\end{array}$ & $13,8 \%$ & $26,20 \%$ & $15,70 \%$ & $25,20 \%$ & $19 \%$ \\
\hline
\end{tabular}

\section{DISCUSIÓN}

Los resultados obtenidos de las personas encuestadas muestran claramente que existe preocupación por la isoapariencia farmacéutica para los profesionales de enfermería, los sujetos encuestados con edades entre 20 a 30 años afirman que es un factor de riesgo para cometer un error de administración.

Los resultados muestran además que existen un mayor número de expertos en la enfermería que aplican la regla de los 5 correctos antes de administrar fármacos erróneamente. Otro factor que se presenta en los resultados con mayor grado de relevancia es la isoapariencia de medicamentos en áreas de emergencia, siendo en algunos casos una de las principales problemáticas para los profesionales de enfermería al momento de suministrar medicación.(Sánchez Ballesteros \& Montero, 2016). Al analizar los resultados del cuestionario realizado los enfermeros/as consideraron que la isoapariencia presente en el envase tiene un impacto negativo para realizar su trabajo y además se consideran peligrosos a los fármacos genéricos con principios activos similares, esto se ve reflejado cómo un porcentaje elevado de los sujetos encuestados que la consideraron un problema.(Sobrecarga, equivocación, falta de capacitación: factores contribuyentes 
en errores de medicación en hospital público chileno, s. f.).Además, los resultados muestran índices medianamente altos en cuanto a la pregunta si algún compañero/a ha administrado un fármaco por su parecido con otro distinto ante la misma pregunta realizada de manera personal, la cual muestra que los profesionales tienen presente siempre realizar una buena administración médica aun cuando exista presencia de isoapariencia en los medicamentos.(Rodríguez et al., 2018) Una medida que podrían optar los hospitales sería prevenir la compra de medicamentos con una apariencia parecida, o también disponer de un sistema para la identificación de características especiales en los envases. (Pérez Ingidua et al., 2020)

Esto sugiere que las consultas sobre errores de medicación deberían realizarse periódicamente de forma anónima. Aunque los distintos centros de salud e incluso los propios hospitales, cuentan con herramientas y medios para conocer esta información, pero por la falta de tiempo, preocupación o poco interés no son realizados por la mayoría de profesionales. Aun utilizando la metodología del queso suizo no se pueden evitar completamente los problemas de isoapariencia en los medicamentos, pero se pudo detectar varios indicios que permitan reducir la problemática, tanto como proponer normativas para adquisición de medicamentos y para el personal en general, los cuales ayudarían directamente al personal médico a tener un mejor desempeño laboral disminuyendo riesgos en las distintas áreas dentro del centro sanitario.(Torres Domínguez, 2005)

\section{CONCLUSIÓN}

Los resultados obtenidos en el estudio muestran una clara preocupación por el problema de la isoapariencia farmacéutica entre los profesionales de Enfermería, ya que un porcentaje elevado de los sujetos encuestados consideró que esta constituye un factor de riesgo para cometer un error de administración.

Se identificó los fármacos genéricos con isoapariencia como los menos adecuados para áreas de emergencia, el mismo que pueden ser peligrosos al tener una mala administración por presencia de isoapariencia en sus envases.(Chaverri Fernández, 2017)

Los resultados muestran complicaciones relacionados a la isoapariencia esto se debe a la falta de información sobre el tema además de factores tales como la similitud de medicamentos existentes en las distintas áreas donde el profesional de salud se desempeña. 
El método del queso suizo ayudo a identificar las posibles causas que se tienen cuando existe isoapariencia en los medicamentos ya que cada rodaja de queso suizo representa una pregunta encuestada si estos hoyos se aliñan el peligro tiene pase directo para que se convierta un accidente por lo cual si generamos barreras y evitamos la isoapariencia podemos limitarlos y permitir que el personal médico pueda realizar de mejor manera su práctica profesional en centros sanitarios.

La necesidad de formación y conocimientos acerca de los errores presentes en la medicación puede disminuir el mismo y ayuda a tener una atención más confiable para el paciente.

Se debe impulsar cada vez más estudios e investigaciones sobre la isoapariencia en áreas de la salud, los cuales pueden ayudar a reducir riesgos y mejorar el servicio que brinda el profesional a sus pacientes.(parte04.pdf, s. f.)

\section{LISTA DE REFERENCIAS}

A systematic literature review on strategies to avoid look-alike errors of labels |

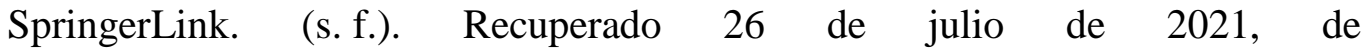
https://link.springer.com/article/10.1007/s00228-018-2471-z

Arguin, A. (s. f.). "¿Ane que? " Caso real de error de medicación por isoapariencia fonética · Stop Errores de Medicación. Stop Errores de Medicación. Recuperado 23 de junio de 2021, de https://www.stoperroresdemedicacion.org/es/blog/aneque-caso-real-de-error-de-medicacion-por-isoapariencia-fonetica/

Cárcamo, A. M. P., Tourinho, F. S. V., \& Alves, T. F. (2020). FACTORES DE RIESGO EN ERRORES DE MEDICACIÓN EN UN HOSPITAL PÚBLICO CHILENO DE ALTA COMPLEJIDADE. Texto \& Contexto - Enfermagem, 29. https://doi.org/10.1590/1980-265X-TCE-2019-0241

Cassius, C., Davis, C. j., Bravard, P., Carre-Gislard, D., Modiano, P., Lebrun-Vignes, B., Ingen-Housz-Oro, S., \& Chosidow, O. (2019). Lookalike and soundalike drugs: A potential cause of cutaneous adverse reactions to drugs. British Journal of Dermatology, 181(3), 626-627. https://doi.org/10.1111/bjd.17842

Chaverri Fernández, J. M. (2017). Detección de errores de medicación en el servicio de emergencias de un hospital privado en Costa Rica: Oportunidades de mejora y seguridad para el paciente. 
Díez, C. P., Campaña, J. M. R., Castro, C. N., Paricio, F. A., Sazatornil, M. R. A., \& Marco, J. B. P. (2017). Errores de medicación en un servicio de urgencias hospitalario: Estudio de situación para mejorar la seguridad de los pacientes. Emergencias: Revista de la Sociedad Española de Medicina de Urgencias y Emergencias, 29(6 (Diciembre)), 412-415.

Errores de medicación en pacientes hospitalizados: Una revisión sistemática. (s. f.). Recuperado 30 de noviembre de 2021, de https://digibug.ugr.es/handle/10481/67696

Feliu, S. (2018, noviembre 3). Infografía: Error humano y queso suizo. Enfermería Nursing. https://asistenciasanitaria.com.ar/2018/11/03/infografia-error-humanoy-queso-suizo/

Ingidua, P., Rivas Paterna, A. B., Portolés Pérez, A., Laredo Velasco, L., \& Vargas Castrillón, E. (2020). La isoapariencia farmacéutica un factor de riesgo de errores de medicación-Metas de Enfermería. Enfermería21. https://www.enfermeria21.com/revistas/metas/articulo/81629/la-isoaparienciafarmaceutica-un-factor-de-riesgo-de-errores-de-medicacion/

ISMP España. (s. f.). Recuperado 11 de noviembre de 2021, de http://www.ismpespana.org/

Isoapariencia para minimizar los errores en la dispensación de medicamentos-ISanidad. (s.f.). Recuperado 23 de junio de 2021, de https://isanidad.com/91870/isoapariencia-para-minimizar-los-errores-en-ladispensacion-de-medicamentos/

Lacasa, C. (Carlos). (2019). Errores de medicación: Evaluación de un sistema de notificación y análisis de los errores en un hospital universitario. https://dadun.unav.edu/handle/10171/58489

Modelo de queso suizo para el análisis de riesgos y fallas. (2019, septiembre 3). Blog de la Calidad. https://blogdelacalidad.com/modelo-de-queso-suizo-para-el-analisisde-riesgos-y-fallas/

Monise, C. (2019, septiembre 3). Modelo de queso suizo para el análisis de riesgos y fallas. Blog de la Calidad. https://blogdelacalidad.com/modelo-de-queso-suizopara-el-analisis-de-riesgos-y-fallas/ 
Montero Delgado, J. A., Plata Paniagua, S., \& Arenas Villafranca, J. J. (2020). El problema de la isoapariencia de medicamentos en España: Es hora de actuar. Journal of Healthcare Quality Research, 35(2), 126-128. https://doi.org/10.1016/j.jhqr.2020.01.003

Mr, C., Jl, S., \& Nr, T. (2016). Riesgo de confusión entre trastuzumab- emtansina y trastuzumab. 3.

Palacio, J., Astier, P., \& Hernández, M. Á. (2017). Medicamentos: Cuando las apariencias engañan. Atención Primaria, 49(7), 375-377. https://doi.org/10.1016/j.aprim.2017.06.002

Palacio Lapuente, J. (2017, junio 9). «Aplicar la isoapariencia es una cuestión de voluntad política»- semFYC. https://www.semfyc.es/isoapariencia-una-cuestion-voluntadpolitica-medicina-seguridad/

Parte04.pdf. (s.f.). Recuperado 30 de noviembre de 2021, de https://www.ispch.cl/newsfarmacovigilancia/07/images/parte04.pdf

Pérez Ingidua, C., Rivas, A., Portoles, A., velasco, leonor, \& Castrillón, emilio. (2020). La isoapariencia farmacéutica un factor de riesgo de errores de medicación. Metas de Enfermería, 23. https://doi.org/10.35667/MetasEnf.2020.23.1003081629

Pérez, M. H. (2019). Errores de medicación en el ámbito hospitalario. 37.

Pinheiro, M. da P., Junior, O. C. da S., Pinheiro, M. da P., \& Junior, O. C. da S. (2017). Evaluación de la cultura de seguridad del paciente en una organización hospitalaria de un hospital universitario. Enfermería Global, 16(45), 309-352. https://doi.org/10.6018/eglobal.16.1.238811

Rodríguez, A. M., Peraza, O. E. C., Fernández, R. D., Gómez, C. M. F., Bermúdez, T. de la P., \& Pérez, A. R. (2018). Los errores de medicación y los valores profesionales en el proceso docente-educativo Cubano. Revista Cubana de Educación Médica Superior, 32(4), 226-239.

Sánchez Ballesteros, P., \& Montero, A. (2016). Los «10 correctos» de enfermería para evitar errores con la medicación. · Stop Errores de Medicación. Stop Errores de Medicación. https://www.stoperroresdemedicacion.org/es/blog/los-10-correctosde-enfermeria-para-evitar-errores-de-medicacion/

SciELO - Brasil-Risk factors for medication errors in the electronic and manual prescription Risk factors for medication errors in the electronic and manual 
prescription. (s.f.). Recuperado 30 de noviembre de 2021, de https://www.scielo.br/j/rlae/a/BNfPG5rLYK7vQ3CBKXCbdCm/?format=html \&lang=es

Sobrecarga, equivocación, falta de capacitación: Factores contribuyentes en errores de medicación en hospital público chileno. (s. f.). Recuperado 30 de noviembre de 2021, de https://scielo.isciii.es/scielo.php?pid=S1132$12962020000200003 \&$ script=sci_arttext\&tlng=en

Suntasig Guaña, L. M. (2015). Análisis de los errores de medicación y sus factores condicionantes en pacientes hospitalizados en los servicios de pediatría y neonatología del hospital "Un Canto a la Vida" en el período comprendido entre los meses de enero a junio del año 2015. Pontificia Universidad Católica del Ecuador. http://repositorio.puce.edu.ec:80/xmlui/handle/22000/9740

Torres Domínguez, A. (2005). Errores en la medicación: Función del farmacéutico. Revista Cubana de Farmacia, 39(2), 1-1.

Tseng, H.-Y., Wen, C.-F., Lee, Y.-L., Jeng, K.-C., \& Chen, P.-L. (2018). Dispensing errors from look-alike drug trade names. European Journal of Hospital Pharmacy, 25(2), 96-99. https://doi.org/10.1136/ejhpharm-2016-001019

Villegas, F., Figueroa-Montero, D., Barbero-Becerra, V., Juárez-Hernández, E., Uribe, M., Chávez-Tapia, N., \& González-Chon, O. (2018). La importancia de la farmacovigilancia intrahospitalaria en la detección oportuna de los errores de medicación. Gaceta Médica de México, 154(2), 172-179. 